\title{
Senior-Centered Design of Health Information Architecture
}

\author{
Panayiotis Zaphiris \\ Institute of Gerontology \\ Wayne State University \\ Detroit, MI 48202 \\ +13135772297 \\ p.zaphiris@wayne.edu
}

\author{
Sri Kurniawan \\ Institute of Gerontology \\ Wayne State University \\ Detroit, MI 48202 \\ +13135772297 \\ s.kurniawan@wayne.edu
}

\begin{abstract}
A series of senior-centered design experiments were used to define the best-fitting web hierarchy for health information for seniors are reported.

Three experiments were performed, namely card sorting, category identification and category labeling. The card sorting experiment's results show that senior participants grouped items conceptually on the higher categorization level but were influenced by literal words found in the link names when grouping items at a finer categorizations deeper down the hierarchy. The category identification and category labeling experiments' results suggests the importance of involving users to properly label the link and group names.
\end{abstract}

\section{Keywords}

Web Hierarchy, Aging, Card Sorting, Health Information, Information Retrieval, senior-centered design.

\section{INTRODUCTION}

\section{Health Information and Aging}

Significant increase of older population has led to various studies investigating the age effect in utilizing the Web as an information resource. Forecasts project that by the year 2030 people aged $65+$ will represent $22 \%$ of US population. Seniors currently representing $13 \%$ of the online users [4]. In 1998, 22 millions individuals reported surfing the Web for medical information, with the number estimated to reach 30 millions by 2000 [1].

Research reviewing medical and health related web sites has found various usability problems. Therefore, it is crucial to make certain that health and medical information are designed in a way that enables even novice users and users with special needs (such as older users or users with disability) to find the information easily and efficiently.

This study attempts to investigate basic components of web sites: the web categories and their hierarchical organization structure $[5,6]$.

\section{Senior-Centered Design}

We propose the term senior-centered design to refer to involving older users in the process of designing products that are targeted towards the aging population.

A more senior-centered approach to designing web site structure requires understanding of seniors' perceived relationship of web site components. One method of collecting this data is through card sorting.

Card sorting is considered as one of the best usability methods for investigating users' mental model of an information space. The resulting tree structures can form the basis for organizing the site and identifying meaningful patterns indicative of general underlying cognitive processes or user mental models [2,3].

\section{METHODOLOGY \\ Participants}

Sixteen seniors aged 55 and above (mean age $=68.69$, SD $=5.97$ ) participated in the card sorting experiment 1 .

A new pool of twenty seniors (mean age $=70.41, \mathrm{SD}=$ 13.41) participated in the category identification follow-up experiment 2. Another new pool of thirteen seniors (mean age $=70.69, \mathrm{SD}=8.18$ ) participated in the category labeling experiment.

All participants live independently in the community (noninstitutionalized) and have no visual and cognitive impairment and functional illiteracy. All participants have at least 13 years of formal education.

\section{Stimulus Material}

The pages used for card sorting were sixty four (64) leaf items taken from the "Health: Aging" hierarchy of Dmoz (http://www.dmoz.org) web site from four main categories, namely: Geriatrics, Diabetes and Alzheimer, Life Cycle and finally Life Expectancy [7].

\section{Apparatus}

3'x5' Index Cards with the Web link names and short descriptions of the content of Web pages were used in the Card Sorting test. USort and EZCalc software by IBM ${ }^{\mathrm{TM}}$ were used for the cluster analysis of card sorting data. 
Paper and pencil questionnaire were used for category identification and category labeling experiments.

\section{Procedure}

\section{Experiment 1}

Each participant was given one set of sixteen (16) index cards from one Dmoz subcategories and was tested in an individual session.

Participants were asked to sort the cards into logical groupings twice. When they felt comfortable with their final sorting arrangement, they were asked to record on paper their card groupings. Participants were asked to list orphan items at the end of their report and were allowed to list items in multiple categories.

After collection the completed sorting data, cluster analysis was performed using EZCalc to produce finalized hierarchical site diagrams [7]

\section{Experiment 2}

A category identification experiment was carried out. Participants were given pages with information from the same main category. Each page consists of short descriptions of the items that were suggested to belong to one group by the previous pool of participants. Each participant was asked to write down a suggested label for the group. The result of this experiment was 3-5 suggested labels per main category.

\section{Experiment 3}

Finally a category labeling experiment was performed using the labels suggested above. Each participant was asked to rank the suggested labels based on their fit to the items in the group (lower number means higher fit). The number is then added up and the label with the smaller sum is the chosen label for the group.

\section{RESULTS AND DISCUSSION}

After plotting the site diagrams [7] can be observed that through the use of user feedback the information structure has transformed from a homogeneous (four items per branch) design obtained from Dmoz to a heterogeneous hierarchy (ranging from two to six items per branch). This is an indication of users' ability to group items in a more meaningful and logical structure that better matches their mental model and resembles existing website link structures.

An other interesting observation is that seniors used in our experiment tended to group items conceptually on the top or grand size of categorization (e.g. grouped items related to Organizations, Diabetes etc) but tended to be influenced by literal words found in link name titles (e.g. "Longevity" or "Anti-aging") when grouping items at finer categorizations deeper down the hierarchy.

Furthermore, unlike in the case of the Dmoz hierarchy where information was often grouped based on geographic location (e.g. Research Institutions in USA versus Research Institutions abroad) our senior users tended to group items based on functionality or service provided (eg. Institutions about Aging, research centers about diabetes).

From category labeling experiment, new names for the proposed categories where obtained. Our hypothesis is that these category labels match the user perception better than the information architecture proposed by Dmoz designers. The validity of this hypothesis needs to be tested by a formal usability experiment.

\section{CONCLUSIONS}

This study intends to apply a series of user-centered design approach to build the information architecture of healthrelated information on the Web for seniors. The results of the study showed that involving prospective users in the design can capture users' underlying perceptions of different components of the information architecture, including the structure and the labels of the hierarchy. The resulting information architecture is expected to be more user-friendly as it is designed based on closer fit to user's mental model.

\section{REFERENCES}

1. Elliott, B. and Elliott, G. High volume medical websites. Del Medical Journal, 72(1), 21-29., 2000.

2. Kanerva, A., Keeker, K., Risden, K., Schuh, E. \& Czerwinski, M. Web Usability at Microsoft Corporation. In J. Ratner, E. Grosse and C. Forsythe (eds.) Human Factors for World Wide Web Development, Lawrence Erlbaum, New York, United States of America, 1997.

3. Nielsen, J., and Sano, D. (1994). SunWeb: User interface design for Sun Microsystem's internal web. Proceedings of the $2^{\text {nd }}$ World Wide Web Conference '94: Mosaic and the Web. Available online at: http://www.ncsa.uiuc.edu/SDG/IT94/Proceedings/HCI Inielsen/sunweb.html

4. U.S. Census Bureau. National Population Projections. Available online at: http://www.census.gov/ population/projections/nation/nas/npas1530.tx

5. Zaphiris, P., Ellis, R.D. Mathematical Modeling of Age Difference in Hierarchical Information Systems, ACM Conference on Universal Usability (Arlington,VA, Nov. 16-17, 2000)

6. Zaphiris, P. Depth vs Breadth in the Arrangement of Web Links, in Proc. of the 44th Annual Meeting of the Human Factors and Ergonomics Society (San Diego, CA, August 2000)

7. Zaphiris, P., Kurniawan, S. Appendix to SeniorCentered Design of Health Information Architecture. Available online at http://pzaphiri.agrino.org/ Papers/CHI2000/ 